\title{
Population Structure Analysis of Globally Diverse Bull Genomes
}

\author{
Neo Christopher Chung ${ }^{1, *}$, Joanna Szyda ${ }^{1}$, Magdalena Frasszczak ${ }^{1}$, and the 1000 Bull \\ Genomes Project ${ }^{2}$
}

\author{
${ }^{1}$ Biostatistics Group, Department of Genetics, Wroclaw University of Environmental and Life Sciences, Wroclaw, \\ 51631, Poland \\ ${ }^{2}$ Membership of the 1000 Bull Genomes Project is provided in the Acknowledgments. \\ *nchchung@gmail.com
}

\begin{abstract}
Since domestication, population bottlenecks, breed formation, and selective breeding have radically shaped the genealogy and genetics of Bos taurus. In turn, characterization of population structure among globally diverse bull genomes enables detailed assessment of genetic resources and origins. By analyzing 432 unrelated bull genomes from 13 breeds and 16 countries, we demonstrate genetic diversity and structural complexity among the global bull population. Importantly, we relaxed a strong assumption of discrete or admixed population, by adapting latent variable models for individual-specific allele frequencies that directly capture a wide range of complex structure from genome-wide genotypes. We identified a highly complex population structure that defies the conventional hypothesis based on discrete membership and contributes to pervasive genetic differentiation in bull genomes. As measured by magnitude of differentiation, selection pressure on SNPs within genes is substantially greater than that on intergenic regions. Additionally, broad regions of chromosome 6 harboring largest genetic differentiation suggest positive selection underlying population structure. We carried out gene set analysis using SNP annotations to identify enriched functional categories such as energy-related processes and multiple development stages. Our comprehensive analysis of bull population structure can support genetic management strategies that capture structural complexity and promote sustainable genetic breadth.
\end{abstract}

\section{Introduction}

Bos taurus (cattle) has long experienced selection for high quality milk and meat production. To maintain and encourage genetic diversity, it is important to characterize the population structure of bulls around the world. Inferring population structure and genetic differentiation play an increasingly important role in conservation efforts, genealogy, and selection programs. In this study, we have analyzed a large number of whole genome sequences of Bos taurus bulls from 13 breeds, representing 16 countries, to characterize population structure and genetic diversity.

Recognizing the importance of cattle genome diversity in genome-wide association studies, genomic predictions, and optimal breeding, there have been substantial efforts to obtain genome-wide genotypes of multiple breeds in diverse geographical locations $^{1-3}$. The 1000 Bull Genomes Consortium has successfully collaborated with institutions from more than 20 countries to collect 1577 whole genome sequences (as of version 5). This international collection of diverse genomes can be regarded as representative of genetic diversity within bulls and thus enables systematic analysis of population genomics. Although the structural complexity of cattle has previously been studied based on limited genome profiles or genetic markers, focusing on regions and breeds ${ }^{4-7}$, a population genetic study involving a large and diverse collection of whole genome sequences has not been performed.

Moreover, most studies assumed discrete structure among representatives of a studied population, as defined by selfidentified breeds. Recent studies using genome-based tree, admixture models, and other techniques demonstrate far greater structural complexity ${ }^{1,2,7}$, but direct estimation and utilization of continuous population structure have been challenging. Logistic factor analysis (LFA) uses recently developed probabilistic models of individual allele frequencies underlying genotypes that are appropriate for a wide range of population structures (e.g., discrete, continuous, or admixture) ${ }^{8}$. Building on principal component analysis (PCA), LFA provides a non-parametric estimation method tailored to genotype data. By modeling each single nucleotide polymorphism (SNP) by the population structure estimated by logistic factors (LFs), genetic differentiation can be directly tested and inferred.

Applying latent variable probabilistic models, we analyzed 432 unrelated Bos taurus genomes from 13 breeds and 16 countries, as part of the 1000 Bull Genomes Project ${ }^{2}$. This study provides detailed assessment of population structure among a diverse panel of whole genome sequences ( $>3.9$ million SNPs per bull). We identified pervasive genetic differentiation 
as suggested by domestication and selection. Through incorporating gene set analyses with genomic features, evolutionary pressure on genetic variation is investigated. Additionally, we present an interactive visualization, which enables exploration of underlying population structure by logistic factors. This study represents one of the first studies in population genomics where potentially inaccurate breeds (or other self-referential subpopulation labels) are intentionally left unused.

\section{Results}

In the 1000 Bull Genomes Project dataset, there were $n=432$ unrelated Bos taurus samples with average sequencing coverage $>5$ (Figure 1). These bulls represent 13 different breeds; namely, Angus, Brown Swiss, Charolais, Gelbvieh, Holstein, Jersey, Limousin, Montbeliard, Normandy, Piedmont, European Red Dairy, Holstein, Red \& White, and Simmental/Fleckvieh. Defined by the official animal identification, our samples came from Australia, Austria, Canada, Denmark, Finland, France, Germany, Italy, Netherlands, New Zealand, Norway, Spain, Sweden, Switzerland, United Kingdom, and United States (Figure 2). Among these genomes, there are $m=3,967,995$ single nucleotide polymorphisms (SNPs) with no missing values and minor allele frequencies $i 0.05$ (Supplementary Fig. 2). To explore structural complexity, whole genome sequences of $n=432$ selected samples were hierarchically clustered using Manhattan distances (Figure 3, colored by 13 different breeds). It is evident that official breed codes (or countries of origin) do not necessarily represent the genetic diversity among bulls represented by SNPs.

The dimension of the population structure in logistic factor analysis was set at $d=7$, as estimated by the VSS algorithm and the scree plot of decreasing eigenvalues (Supplementary Fig. 3). The estimated logistic factors demonstrate the genetic continuum, reflecting shared origins of genetics and overlapping goals of breeding programs since domestication (Figure 4). At the same time, the logistic factor 4 displays a clear distinction of Brown Swiss (from Switzerland, Germany, France, and Italy) and projection of logistic factors allows straightforward visual identification of clusters. We enable interactive exploration of this population structure by creating an online app visualizing logistic factors according to user-specified parameters (https://nnnn.shinyapps.io/bullstructure/).

We discovered diverse and pervasive genetic differentiation with respect to the population structure of bulls. When applying the resampling-based jackstraw method to test association between SNPs and logistic factors, we observed that the vast majority of SNPs are statistically significantly differentiated (an estimated proportion of null SNPs $\widehat{\pi}_{0}$ of $6.2 \%$ ). A proportion of variation explained by $d=7 \mathrm{LFs}$ for each SNP is approximated by McFadden's pseudo $R^{2}$. We found that the median and mean values of McFadden's pseudo $R^{2}$ are 0.070 and 0.087 , respectively (Figure 5). The chromosome 6 contained substantially more SNPs with high $R^{2}$ than other chromosomes; it harbors 166 (39.0\%) out of 426 SNPs with $R^{2}>0.6$, as well as all $29(100 \%)$ SNPs with $R^{2}>0.7$. On the other hand, the $\mathrm{X}$ chromosome shows the least variation with respect to logistic factors, containing zero SNP with $R^{2}>0.5$. The top 1000 genomic features that are associated with differentiated SNPs are shown in Supplementary Data 1 . Note that we additionally conducted an independent robustness analysis with $d=22$ logistic factors (as suggested by a cross-validation method), which confirm highly consistent genetic differentiation, with an overall $R^{2}$ correlation of 0.93 .

Among SNPs with the highest $R^{2}>0.7$, there exist two tight groups on chromosome 6; specifically 14 SNPs (13 within 50kbp of known genomic features) positioned between 71101370 and 71600122 and 15 SNPs (11 within 50kbp of known genomic features) positioned between 38482423 and 39140537. 83\% of those most differentiated SNPs (20 out of 24 SNPs with known genomic features) are within or close to genes related to the selection sweep according to ${ }^{9}$. Among the first group, five SNPs fall within CHIC2 (ENSBTAG00000032660), while the closest features within 50kbp also include GSX2 (ENSBTAG00000045812), U6 spliceosomal RNA (ENSBTAG00000042948), and novel pseudogene (ENSBTAG00000004082). U6 spliceosomal RNA (ENSBTAG00000042948) and novel pseudogene (ENSBTAG00000004082) are known to be associated with milk protein percentage ${ }^{10}$. In the second group, the exact overlaps occur in FAM184B (ENSBTAG00000005932), LCORL (ENSBTAG00000046561), and NCAPG (ENSBTAG00000021582). LCORL encodes a transcription factor whose human ortholog is involved in spermatogenesis, whereas NCAPG is crucial in mitosis and meiosis. Expecting much granular investigation of such genomic features, the list of 396,800 SNPs at the top 90 percentile $\left(R^{2}>.174\right)$ is available as Supplementary Data 2.

To better understand evolutionary and biological processes, we conducted gene set analyses using genomic annotations of SNPs. Firstly, we found that SNPs located within known genomic features have about $1.8 \%$ higher $R^{2}$ measures than intergenic SNPs without annotations (MWW p-values $9.85 \times 10^{-106}$ ). On the other hand, among intergenic SNPs, we found no significant correlation (p-value of 0.44) between SNP-feature distances and $R^{2}$ measures (Supplementary Fig. 4). Secondly, among genic SNPs, $R^{2}$ measures corresponding to SNPs within exons are slightly higher than those within introns by $0.27 \%$ with a MWW p-value $3.89 \times 10^{-29}$. Start/stop codons and 3'/5' UTR do not exhibit statistically significant difference from other genic SNPs. Lastly, we used 338 genes that are closest to SNPs with $R^{2}>0.5$ in the DAVID functional annotation tools. We found a total of 34 enriched annotation clusters, of which 11 clusters with enrichment scores $>0.5$ are shown in (Table 1). Biological processes and functions related to calcium-binding domain (cluster 1 and 9) and iron containing hemeproteins related to ATP (cluster 3 and 6) exhibit strong enrichment, potentially reflecting causes of population structure. Notably, we observed functional clusters for sexual, respiratory, and embryonic developments (cluster 5, 7, and 10, respectively). 


\section{Discussion}

Bos taurus has played a crucial role in ancient and modern societies alike by providing agricultural support and essential nutrients. Accurate characterization of its population structure helps conservation of genetic resources and optimal selection programs, ensuring a healthy and sustainable cattle population. In this process, we can better infer the genetic and functional variation that underlies the population structure. Our study, using 432 whole genome sequences of unrelated Bos taurus samples, provides a comprehensive and rigorous assessment of population structure among diverse bulls.

Assumptions underlying population structure and its estimation methods based on genotypes have evolved to address growing genomics data in terms of complexity and scales ${ }^{11-13}$. Particularly, the contentious and ambiguous definition of breeds merely requires "certain distinguishable characteristics" and practically relies on self-referential breed registry for sire and dam ${ }^{14}$. Therefore, we have relaxed strong assumptions often used in population genomics, by employing latent variable probabilistic models ${ }^{8}$. In this study, we did not assume discrete or admixed populations. The present framework can nonetheless capture a broad range of arbitrarily complex structure including the aforementioned configurations. Based on observing a largely continuous genetic spectrum compared to breeds, we demonstrated that breeds do not account for structural complexity. We speculate that many cattle breeds, including presumed founders, are not as isolated or discrete as one would be led to believe. The population structure of our bull genomes in 7-dimensional logistic factors can be explored on our interactive visualization website.

When modeling SNPs with logistic factors in generalized linear models, we found widespread genetic differentiation due to population structure. This likely arose from a long history of breed formation and artificial selection, such that different national breeding programs may have caused weak and pervasive systematic variation. Despite being blind to breeds, the majority of the most differentiated SNPs in our study have been identified as under selection sweep. The chromosome 6 harboring a large proportion of highly differentiated SNPs has been suggested for strong selective sweep ${ }^{1}$, and may also be associated with calving ease and carcass weight ${ }^{15,16}$. Interestingly, given that the novel pseudogene (ENSBTAG00000004082), which has been known to be associated with calving performance ${ }^{17}$ and protein percentage ${ }^{10}$ is strongly associated with population structure, we suspect that it plays a crucial functional role in bull genomes. Overall, our genome-wide study of differentiation suggests stronger evolutionary pressure on genic regions. Enrichment analysis of genome annotations provides strong indications that functional groups related to energy production and development stages underlie the systematic variation in the panel of diverse bulls.

Pedigrees were used to remove $72.6 \%$ of bull samples related by progenitors, resulting in a panel of 432 genomes analyzed. However, undocumented kinship may potentially bias our population structure analysis, just as it does other methods that utilize breed and other subpopulation information. We advocate for stronger linkage between breeding programs and registries. The structural complexity among bull genomes discovered in this study can be used directly to identify genetic association with quantitative traits ${ }^{18}$. However, the 1000 Bull Genomes Consortium does not collect quantitative traits as its main goal is comprehensive identification of genomic variants. Lastly, the 1000 Bull Genomes Project, which is among the largest collections in this area of study, is still lacking samples from Asia, Africa, and South America.

This study paves a way to further our understanding of genetic diversity among modern cattle breeds. Our identification of systematic genetic differentiation may inform conservation efforts to preserve heritage breeds and maintain genetic diversity. Considering our flexible assumption about population structure and exclusive use of whole genome sequences, our highly differentiated SNPs, gene set analysis, and functional enrichment show how we can dispense of potentially inaccurate subpopulation labels in population genomics.

\section{Methods}

\section{Bull Genomes}

The 1000 Bull Genomes Project has collaborated with worldwide institutions to gather whole-genome sequences of diverse breeds. Its initial efforts have vastly expanded known single nucleotide polymorphisms (SNPs) and copy number variations (CNVs) in Bos taurus ${ }^{2}$. Currently, it covers 1577 bull samples as of version 5 released in 2015, among which 1507 and 70 bull genomes were sequenced with Illumina/Solexa and ABI SOLiD technology, respectively. For analysis of population structure, we selected unrelated bulls with average sequencing coverage greater than 5. Among sibs only one representative was selected randomly. SNP genotypes were identified prior to our study based on whole genome sequence data of bulls, using a multi-sample variant calling procedure. Polymorphisms with minor allele frequencies below 0.05 were removed from analyses. For processing whole-genome sequences, we used vcftools v0.1.14 ${ }^{19}$, BEDOPS v2.4.15 ${ }^{20}$, and R v3.2.2 ${ }^{21}$.

\section{Statistical Analysis}

To infer population structure directly from a genome-wide genotype matrix, we consider a probabilistic model of individual allele frequencies. In particular, by using logistic factor analysis ${ }^{8}$ that captures systematic variation of individual-specific allele 
frequencies arising from discrete or continuous sub-population, spatial variation, admixture, and other structures, we relax statistical assumptions imposed on bulls by its official breed and country code defined in the animal registration ID. While the statistical models and algorithms are extensively described in elsewhere ${ }^{8}$, we provide a brief overview of this approach here.

Consider a genotype matrix $\mathbf{Y}$ with $m$ SNPs and $n$ bulls. For each $y_{i j}$, an individual-specific allele frequency for $i^{\text {th }}$ SNP and $j^{\text {th }}$ bull is $f_{i j} \in[0,1]$. This collection of parameters (a $m \times n \mathbf{F}$ matrix) is transformed into real numbers via the logit function, which allows computation of the underlying latent structure. Overall, the statistical model considered is

$$
\operatorname{logit}(\mathbf{F})=\mathbf{A H} \text {. }
$$

Then, the population structure is captured by $d$ logistic factors (LFs) $\mathbf{H}$ which can be estimated by applying principal component analysis (PCA) to $\operatorname{logit}(\mathbf{F})$. Note that $\mathbf{A}$ is a matrix of coefficients in a logistic regression. The dimensions of logistic factors are estimated by comparing the observed correlation matrix to a series of hypothesized structures derived from selected variables of large loadings ${ }^{22}$. In the Very Simple Structure (VSS) algorithm, we considered $d=1, \ldots, 100$, while applying principal component analysis on the mean-centered genotypes (R package psych). Eigenvalues of $m^{-1} \mathbf{Y}^{T} \mathbf{Y}$ and percent variance explained by each component are visually inspected for the inflection point (e.g., elbow). For robustness analysis to confirm genetic differentiation, we alternatively used cross-validation approximations to choose $d^{23}$.

To investigate genetic differentiation with respect to the population structure, we test association between $i^{\text {th }}$ SNP $\mathbf{y}_{i}$ and estimate logistic factors $\widehat{\mathbf{H}}$. We model SNPs with $d$ logistic factors in a logistic regression (with a logit link function), where the deviance statistic compares the full (LFs) model $\mathbf{Y} \sim \widehat{\mathbf{H}}$ and the null (intercept) model $\mathbf{Y} \sim \mathbf{1}^{24}$. We take account of the fact that the population structure is directly estimated from $\mathbf{Y}$ by utilizing the resampling-based jackstraw method ${ }^{25}$. For each of $B$ iterations, the jackstraw method introduces a small number $s \ll m$ of permuted SNPs under a null model $\mathbf{Y} \sim \mathbf{1}$ and computes $s$ empirical null deviance statistics. P-values are calculated by ranking observed deviances with an empirical distribution of $B \times s$ deviances, as adapted from the resampling-based jackstraw approach ${ }^{25}$. This method based on a logistic regression is implemented in the jackstraw V1.1 package, freely available on the Comprehensive R Archive Network (https://cran.r-project.org/web/packages/jackstraw). A proportion of SNPs that are not associated with LFs $\left(\pi_{0}\right)$ is then estimated from $m$ p-values.

To approximate how much of the variation in genotypes is explained by the population structure, we calculate McFadden's pseudo $R^{2}$ that is appropriate for a logistic regression ${ }^{26}$. This methodology is operationally similar to detecting genomic signatures with PCA ${ }^{27}$, although the difference arises from directly modeling categorical SNP data. For $i^{\text {th }}$ SNP,

$$
R_{i}^{2}=1-\frac{\log \left(L_{i}^{\text {full }}\right)}{\log \left(L_{i}^{\text {null }}\right)}
$$

where $\log \left(L_{i}^{\text {full }}\right)$ and $\log \left(L_{i}^{\text {null }}\right)$ are maximum $\log$-likelihoods of the full and null models, respectively. As this study only considers McFadden's pseudo $R^{2}$ in logistic regressions, we will henceforth refer to it as $R^{2}$ when clear in context.

\section{Annotation and Enrichment}

For genome annotation, we used the latest Bos taurus reference genome from the Center for Bioinformatics and Computational Biology, University of Maryland (downloaded from the NCBI server ftp://ftp.ncbi.nlm.nih.gov/, version UMD3.1.83).

When testing whether the distribution of McFadden's pseudo $R^{2}$ measures are significantly different according to feature types, we used the Mann-Whitney-Wilcoxon (MWW) test ${ }^{28}$. With a large sample size, a Normal approximation is used to compute MWW p-values. In particular, we investigated whether SNPs falling within genes may have a higher McFadden's pseudo $R^{2}$ than those in intergenic regions. Among SNPs with known feature assignments, MWW tests were used to infer if a particular feature type is associated with significantly higher $R^{2}$ measures.

Lastly, because some of SNPs are in intergenic regions with no known annotations, we utilized the closest features function

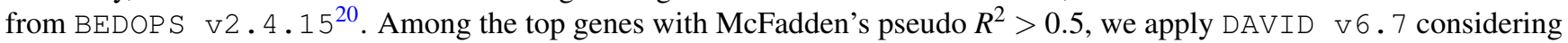
GO, KEGG pathways, InterPro, SwissProt Protein Information Resource, and other databases to identify enrichment of biological processes and functional pathways ${ }^{29}$. For intergenic SNPs, we searched the reference genome for the closest genes, which were used in DAVID v6.7. When clustering functional annotations, we set "Classification Stringency" to high.

\section{References}

1. Gibbs, R. A. et al. Genome-wide survey of SNP variation uncovers the genetic structure of cattle breeds. Science 324, 528-532 (2009). URL http://dx.doi.org/10.1126/science.1167936.

2. Daetwyler, H. D. et al. Whole-genome sequencing of 234 bulls facilitates mapping of monogenic and complex traits in cattle. Nature Genetics 46, 858-865 (2014). URL http: / / dx. doi .org/10.1038/ng.3034. 
3. Stothard, P. et al. A large and diverse collection of bovine genome sequences from the canadian cattle genome project. GigaScience 4 (2015). URL http: / / dx. doi .org/10.1186/s13742-015-0090-5.

4. Troy, C. S. et al. Genetic evidence for near-eastern origins of european cattle. Nature 410, 1088-1091 (2001). URL http://dx.doi.org/10.1038/35074088.

5. Zenger, K. R., Khatkar, M. S., Cavanagh, J. A. L., Hawken, R. J. \& Raadsma, H. W. Genome-wide genetic diversity of holstein friesian cattle reveals new insights into Australian and global population variability, including impact of selection. Animal Genetics 38,7-14 (2007). URL http://dx.doi.org/10.1111/j.1365-2052.2006.01543.x.

6. McKay, S. D. et al. An assessment of population structure in eight breeds of cattle using a whole genome SNP panel. BMC Genet 9, 37 (2008). URL http://dx.doi.org/10.1186/1471-2156-9-37.

7. Jemaa, S. B., Boussaha, M., Mehdi, M. B., Lee, J. H. \& Lee, S.-H. Genome-wide insights into population structure and genetic history of tunisian local cattle using the illumina bovinesnp50 beadchip. BMC Genomics 16 (2015). URL http://dx.doi.org/10.1186/s12864-015-1638-6.

8. Hao, W., Song, M. \& Storey, J. D. Probabilistic models of genetic variation in structured populations applied to global human studies. Bioinformatics btv641 (2015). URL http://dx.doi.org/10.1093/bioinformatics/btv641.

9. Gutierrez-Gil, B., Arranz, J. J. \& Wiener, P. An interpretive review of selective sweep studies in bos taurus cattle populations: identification of unique and shared selection signals across breeds. Front. Genet. 6 (2015). URL http: //dx.doi.org/10.3389/fgene.2015.00167.

10. Meredith, B. K. et al. Genome-wide associations for milk production and somatic cell score in holstein-friesian cattle in Ireland. BMC Genet 13, 21 (2012). URL http://dx.doi.org/10.1186/1471-2156-13-21.

11. Balding, D. J. \& Nichols, R. A. A method for quantifying differentiation between populations at multi-allelic loci and its implications for investigating identity and paternity. Genetica 96, 3-12 (1995).

12. Pritchard, J. K., Stephens, M. \& Donnelly, P. Inference of population structure using multilocus genotype data. Genetics 155, 945-959 (2000).

13. Novembre, J. \& Stephens, M. Interpreting principal component analyses of spatial population genetic variation. Nat Genet 40, 646-649 (2008). URL http: / / dx. doi.org/10.1038/ng.139.

14. Briggs, H. M. \& Briggs, D. M. Modern breeds of livestock (Macmillan, 1980), 4 edn.

15. Bongiorni, S., Mancini, G., Chillemi, G., Pariset, L. \& Valentini, A. Identification of a short region on chromosome 6 Affecting direct calving ease in piedmontese cattle breed. PLoS ONE 7, e50137 (2012). URL http: / / dx . doi . org/ 10.1371 / journal.pone.0050137.

16. Setoguchi, K. et al. Cross-breed comparisons identified a critical 591-kb region for bovine carcass weight QTL (CW-2) on chromosome 6 and the ile-442-met substitution in NCAPG as a positional candidate. BMC Genet 10, 43 (2009). URL http://dx.doi.org/10.1186/1471-2156-10-43.

17. Purfield, D. C., Bradley, D. G., Evans, R. D., Kearney, F. J. \& Berry, D. P. Genome-wide association study for calving performance using high-density genotypes in dairy and beef cattle. Genetics Selection Evolution 47 (2015). URL http://dx.doi.org/10.1186/s12711-015-0126-4.

18. Song, M., Hao, W. \& Storey, J. D. Testing for genetic associations in arbitrarily structured populations. Nature Genetics 47, 550-554 (2015). URL http://dx.doi.org/10.1038/ng.3244.

19. Danecek, P. et al. The variant call format and VCFtools. Bioinformatics 27, 2156-2158 (2011). URL http: //dx. doi. org/10.1093/bioinformatics/btr330.

20. Neph, S. et al. BEDOPS: high-performance genomic feature operations. Bioinformatics 28, 1919-1920 (2012). URL http://dx.doi.org/10.1093/bioinformatics/bts277.

21. R Core Team. R: A Language and Environment for Statistical Computing. R Foundation for Statistical Computing, Vienna, Austria (2015). URL https: / /www.R-project.org/.

22. Revelle, W. \& Rocklin, T. Very simple structure: An alternative procedure for estimating the optimal number of interpretable factors. Multivariate Behavioral Research 14, 403-414 (1979).

23. Josse, J. \& Husson, F. Selecting the number of components in principal component analysis using cross-validation approximations. Computational Statistics \& Data Analysis 56, 1869-1879 (2012). URL http://dx. doi . org/10 . 1016/j.csda.2011.11.012.

24. Hosmer Jr, D. W., Lemeshow, S. \& Sturdivant, R. X. Applied logistic regression (Wiley, 2013). 
25. Chung, N. C. \& Storey, J. D. Statistical significance of variables driving systematic variation in high-dimensional data. Bioinformatics 31, 545-554 (2015).

26. McFadden, D. Conditional logit analysis of qualitative choice behavior. In Zarembka, P. (ed.) FRONTIERS IN ECONOMETRICS, 105-142 (Academic Press, New York, 1974).

27. Duforet-Frebourg, N., Luu, K., Laval, G., Bazin, E. \& Blum, M. G. Detecting genomic signatures of natural selection with principal component analysis: Application to the 1000 genomes data. Mol Biol Evol 33, 1082-1093 (2015). URL http://dx.doi.org/10.1093/molbev/msv334.

28. Mann, H. B. \& Whitney, D. R. On a test of whether one of two random variables is stochastically larger than the other. Ann. Math. Statist. 18, 50-60 (1947). URL http://dx.doi.org/10.1214/aoms/1177730491.

29. Huang, D. W., Sherman, B. T. \& Lempicki, R. A. Systematic and integrative analysis of large gene lists using DAVID bioinformatics resources. Nat Protoc 4, 44-57 (2008). URL http: / / dx. doi .org/10.1038/nprot . 2008.211.

\section{Acknowledgements}

This work was supported by grant Polish National Science Centre (NCN) grant 2014 / 13 / B / NZ9 / 02016. Part of data storage and computation were carried out at the Poznan Supercomputing and Networking Centre. N.C.C. was supported by the Leading National Research Center Programme 04/KNOW2/2014. The membership of the 1000 Bull Genomes Project are: Hans Rudolf Fries, Mogens Sand $\varnothing$ Lund, Bernt Guldbrandtsen, Didier Boichard, Paul Stothard, Roel Veerkamp, Michael Goddard, Curtis P Van Tassell, and Ben Hayes.

\section{Author contributions statement}

N.C.C. conceived the study, analyzed data, wrote the first draft. M.F. contributed to editing the data. N.C.C. and J.S. revised the manuscript and contribute to the discussion.

\section{Additional information}

N.C.C., M.F., J.S., and the 1000 Bull Genomes Project have no competing financial interest. 


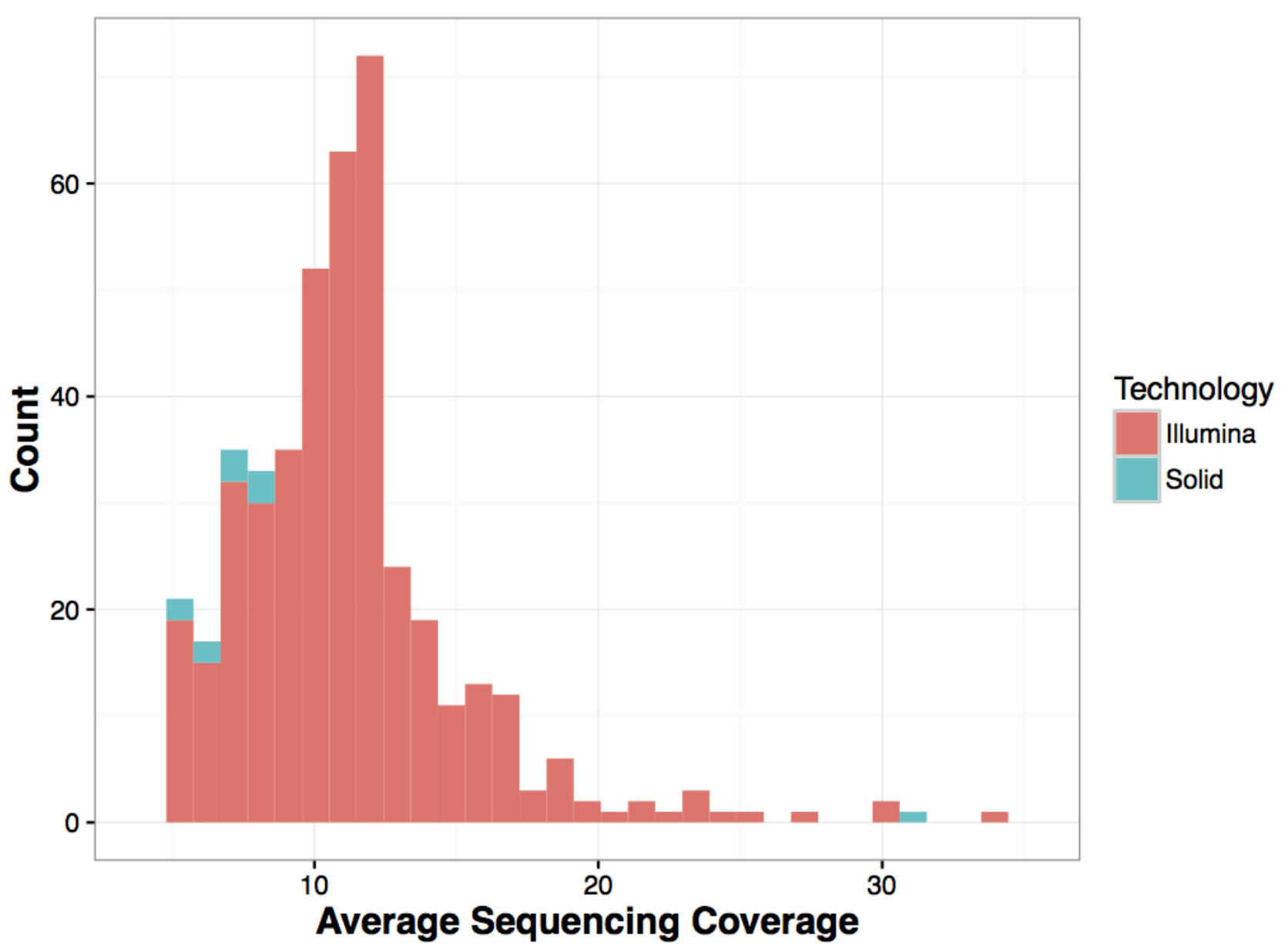

Figure 1. Average sequencing coverage of 432 bull samples.

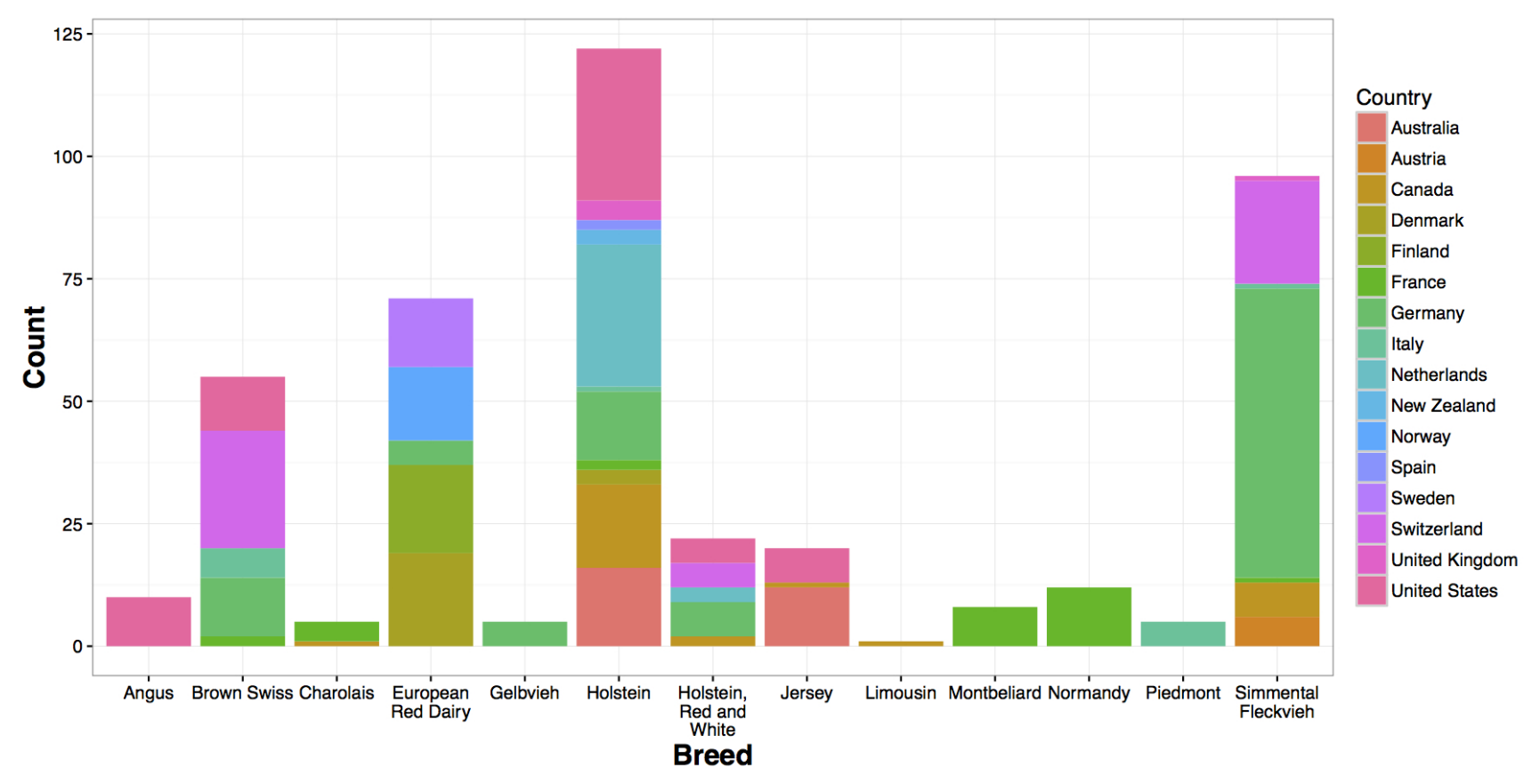

Figure 2. Bar plot of cattle breeds, with a number of samples colored by countries of origin. 
bioRxiv preprint doi: https://doi.org/10.1101/059139; this version posted June 15, 2016. The copyright holder for this preprint (which was not certified by peer review) is the author/funder, who has granted bioRxiv a license to display the preprint in perpetuity. It is made available under aCC-BY-NC-ND 4.0 International license.

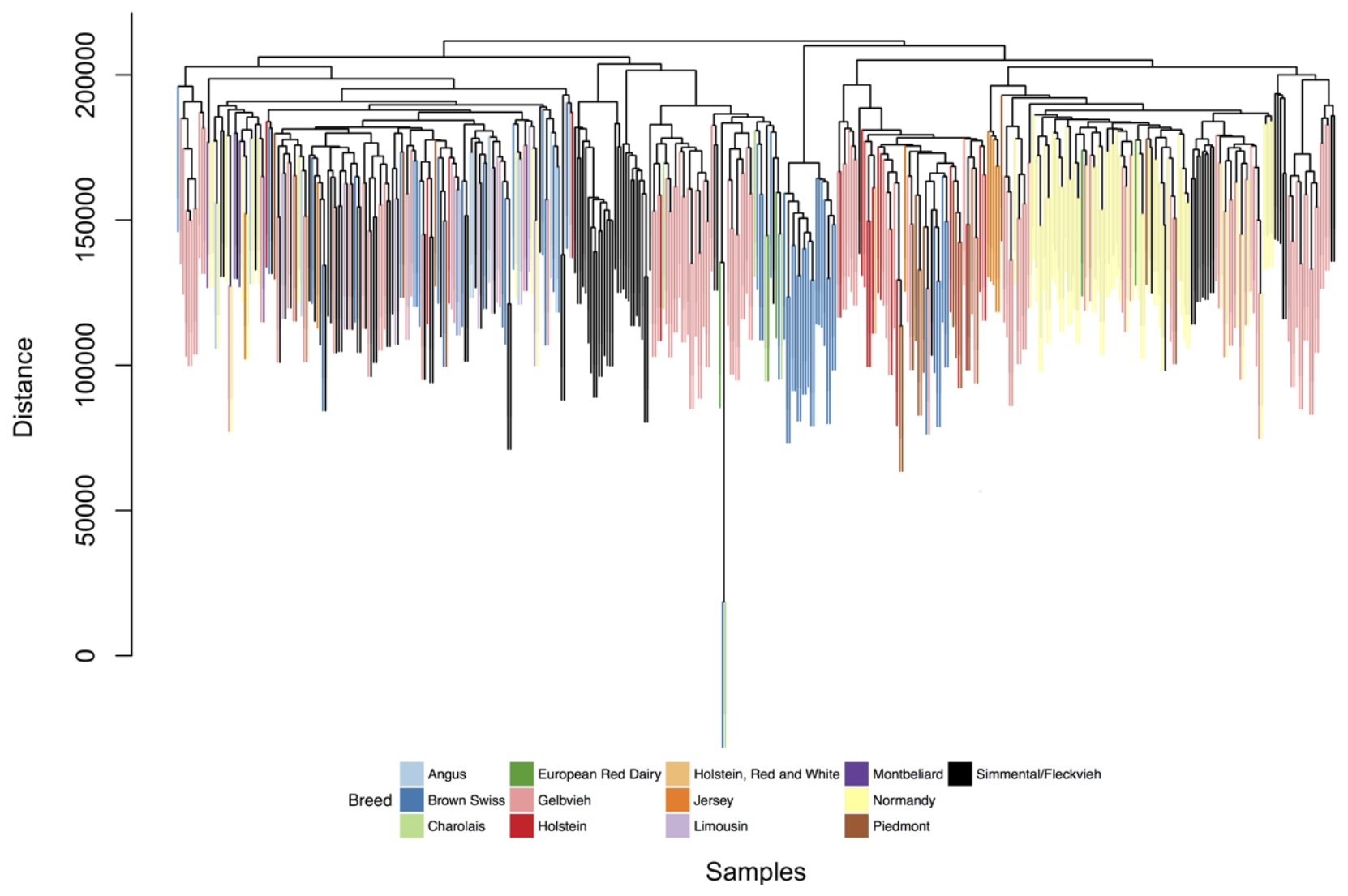

Figure 3. Hierarchical clustering of 432 bull genomes. Genome-wide SNPs are clustered using Manhattan distances and samples are colored by breeds. 


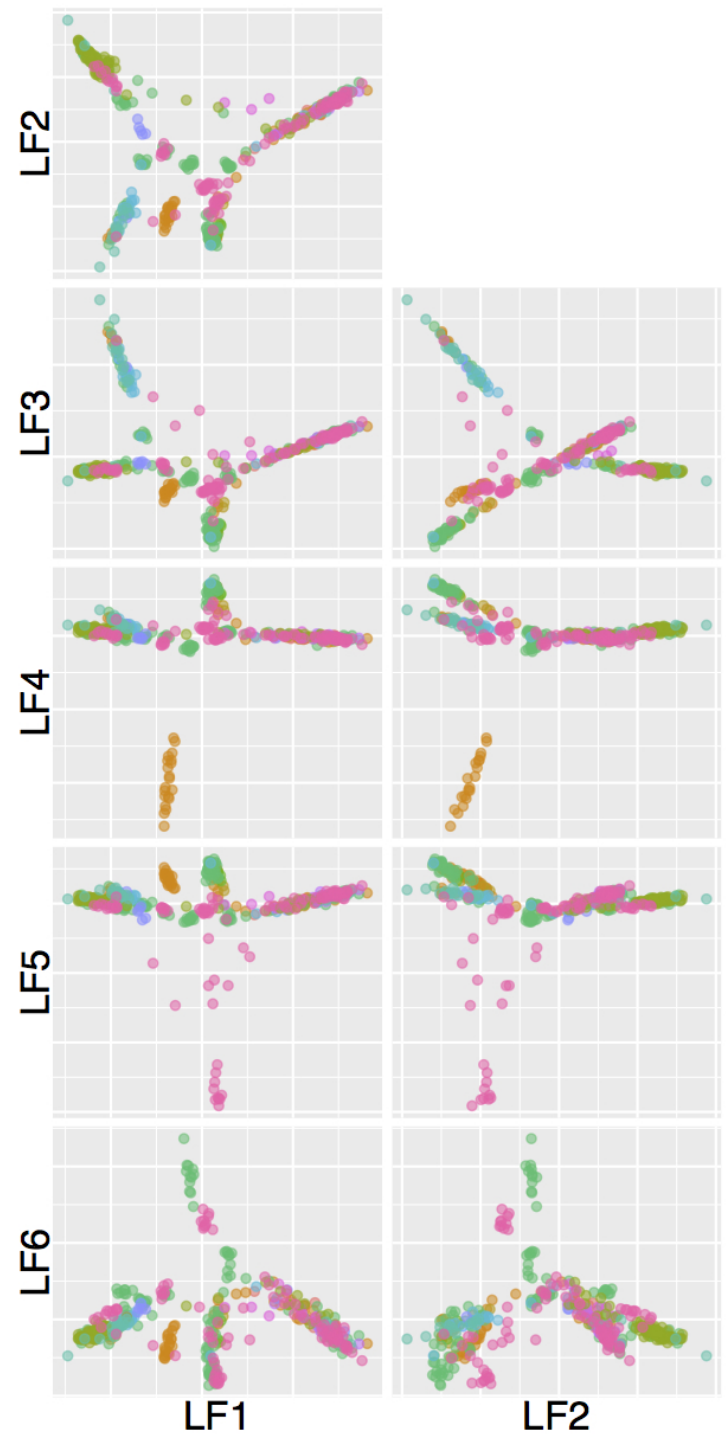

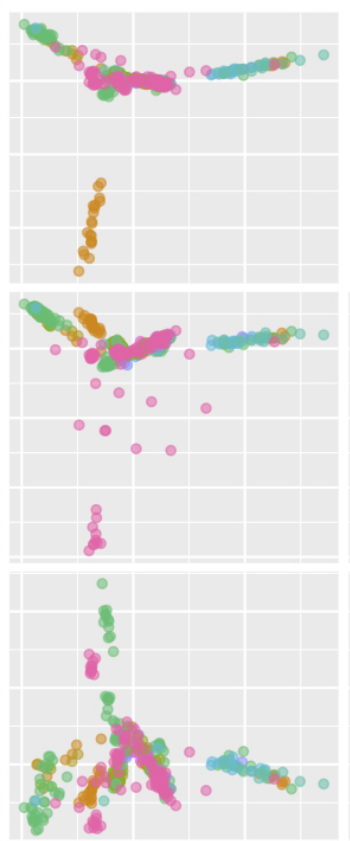

LF3

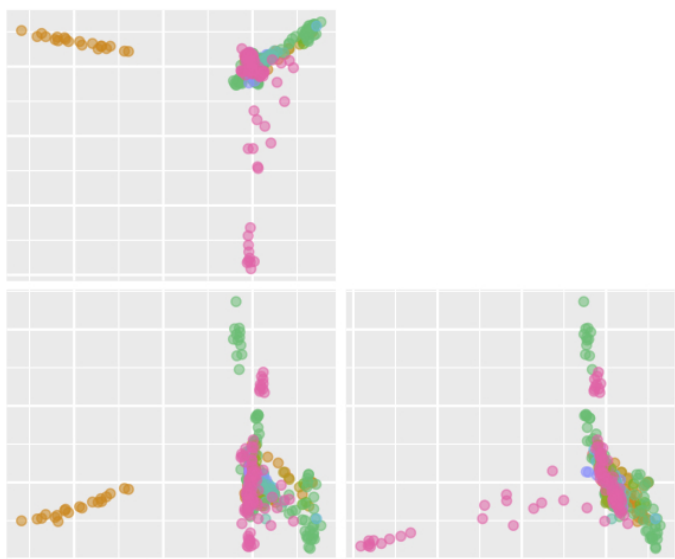

LF4

\section{LF5}

Figure 4. Scatterplots of logistic factors (LFs). All pairs of $6 \mathrm{LFs}$ are plotted, excluding the intercept term. Data points corresponding to 432 bull genomes are colored by 13 breeds. Interactive visualization available at https://nnnn. shinyapps.io/bullstructure/. 
bioRxiv preprint doi: https://doi.org/10.1101/059139; this version posted June 15,2016 . The copyright holder for this preprint (which was not certified by peer review) is the author/funder, who has granted bioRxiv a license to display the preprint in perpetuity. It is made available under aCC-BY-NC-ND 4.0 International license.

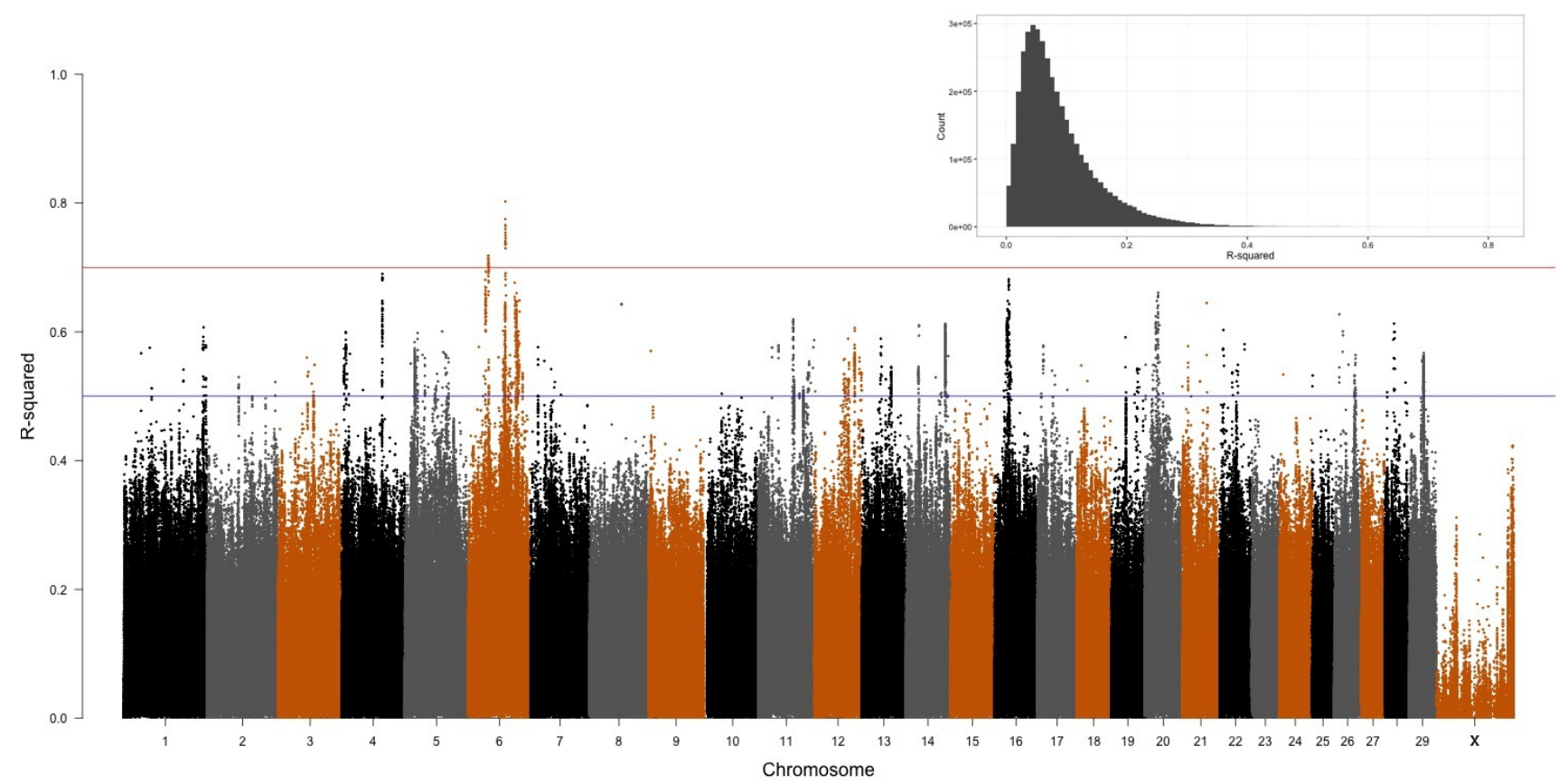

Figure 5. Genome-wide pseudo $R^{2}$ measures with respect to logistic factors (LFs). The distribution is highly skewed towards 0 , which leads to overplotting in a low range (see an insert for a genome-wide histogram). Overall, the median and mean are 0.070 and 0.087 , respectively 
bioRxiv preprint doi: https://doi.org/10.1101/059139; this version posted June 15, 2016. The copyright holder for this preprint (which was not certified by peer review) is the author/funder, who has granted bioRxiv a license to display the preprint in perpetuity. It is made available under aCC-BY-NC-ND 4.0 International license.

Table 1. Enriched functional clusters, for genes associated with $R^{2}>0.5$

\begin{tabular}{|c|c|c|c|c|}
\hline Cluster 1 & Enrichment Score: 1.405 & Calcium-binding domain & & \\
\hline Category & Term & Count & $\%$ & PValue \\
\hline INTERPRO & IPR018247:EF-HAND 1 & 6 & 2.098 & 0.035 \\
\hline INTERPRO & IPR018249:EF-HAND 2 & 6 & 2.098 & 0.038 \\
\hline INTERPRO & IPR011992:EF-Hand type & 6 & 2.098 & 0.045 \\
\hline Cluster 2 & Enrichment Score: 1.372 & Cysteine-type activity & & \\
\hline Category & Term & Count & $\%$ & PValue \\
\hline GOTERM_MF_FAT & GO:0004198 calcium-dependent cysteine-type endopeptidase activity & 3 & 1.049 & 0.011 \\
\hline GOTERM_MF_FAT & GO:0008234 cysteine-type peptidase activity & 4 & 1.399 & 0.066 \\
\hline GOTERM_MF_FAT & GO:0004197 cysteine-type endopeptidase activity & 3 & 1.049 & 0.106 \\
\hline Cluster 3 & Enrichment Score: 0.897 & Cytochrome & & \\
\hline Category & Term & Count & $\%$ & PValue \\
\hline PIR_SUPERFAMILY & PIRSF000045:cytochrome P450 CYP2D6 & 3 & 1.049 & 0.013 \\
\hline INTERPRO & IPR002401:Cytochrome P450, E-class, group I & 3 & 1.049 & 0.068 \\
\hline INTERPRO & IPR017973:Cytochrome P450, C-terminal region & 3 & 1.049 & 0.080 \\
\hline INTERPRO & IPR017972:Cytochrome P450, conserved site & 3 & 1.049 & 0.084 \\
\hline SP_PIR_KEYWORDS & heme & 4 & 1.399 & 0.091 \\
\hline INTERPRO & IPR001128:Cytochrome P450 & 3 & 1.049 & 0.107 \\
\hline SP_PIR_KEYWORDS & Monooxygenase & 3 & 1.049 & 0.124 \\
\hline COG_ONTOLOGY & Secondary metabolites biosynthesis, transport, and catabolism & 3 & 1.049 & 0.148 \\
\hline GOTERM_MF_FAT & GO:0020037 heme binding & 4 & 1.399 & 0.159 \\
\hline GOTERM_MF_FAT & GO:0046906 tetrapyrrole binding & 4 & 1.399 & 0.176 \\
\hline GOTERM_MF_FAT & GO:0009055 electron carrier activity & 4 & 1.399 & 0.301 \\
\hline SP_PIR_KEYWORDS & iron & 4 & 1.399 & 0.399 \\
\hline GOTERM_MF_FAT & GO:0005506 iron ion binding & 4 & 1.399 & 0.614 \\
\hline Cluster 4 & Enrichment Score: 0.860 & Signaling & & \\
\hline Category & Term & Count & $\%$ & PValue \\
\hline UP_SEQ_FEATURE & signal peptide & 19 & 6.643 & 0.048 \\
\hline SP_PIR_KEYWORDS & signal & 19 & 6.643 & 0.111 \\
\hline SP_PIR_KEYWORDS & glycoprotein & 16 & 5.594 & 0.492 \\
\hline Cluster 5 & Enrichment Score: $\mathbf{0 . 8 3 3}$ & Sexual development & & \\
\hline Category & Term & Count & $\%$ & PValue \\
\hline GOTERM_BP_FAT & GO:0045137 development of primary sexual characteristics & 3 & 1.049 & 0.117 \\
\hline GOTERM_BP_FAT & GO:0003006 reproductive developmental process & 4 & 1.399 & 0.151 \\
\hline GOTERM_BP_FAT & GO:0007548 sex differentiation & 3 & 1.049 & 0.180 \\
\hline Cluster 6 & Enrichment Score: 0.760 & Ion binding & & \\
\hline Category & Term & Count & $\%$ & PValue \\
\hline GOTERM_MF_FAT & GO:0043167 ion binding & 40 & 13.986 & 0.130 \\
\hline GOTERM_MF_FAT & GO:0046872 metal ion binding & 38 & 13.287 & 0.190 \\
\hline GOTERM_MF_FAT & GO:0043169 cation binding & 38 & 13.287 & 0.213 \\
\hline Cluster 7 & Enrichment Score: 0.725 & Respiratory development & & \\
\hline Category & Term & Count & $\%$ & PValue \\
\hline GOTERM_BP_FAT & GO:0030324 lung development & 3 & 1.049 & 0.145 \\
\hline GOTERM_BP_FAT & GO:0030323 $\sim$ respiratory tube development & 3 & 1.049 & 0.145 \\
\hline GOTERM_BP_FAT & GO:0060541 respiratory system development & 3 & 1.049 & 0.150 \\
\hline GOTERM_BP_FAT & GO:0035295 tube development & 3 & 1.049 & 0.400 \\
\hline Cluster 8 & Enrichment Score: $\mathbf{0 . 7 2 3}$ & Protease activity & & \\
\hline Category & Term & Count & $\%$ & PValue \\
\hline GOTERM_MF_FAT & GO:0004175 endopeptidase activity & 8 & 2.797 & 0.129 \\
\hline GOTERM_MF_FAT & GO:0070011 peptidase activity, acting on L-amino acid peptides & 9 & 3.147 & 0.190 \\
\hline GOTERM_MF_FAT & GO:0008233 peptidase activity & 9 & 3.147 & 0.215 \\
\hline GOTERM_BP_FAT & GO:0006508 proteolysis & 12 & 4.196 & 0.242 \\
\hline Cluster 9 & Enrichment Score: $\mathbf{0 . 7 0 3}$ & Calcium-binding domain & & \\
\hline Category & Term & Count & $\%$ & PValue \\
\hline UP_SEQ_FEATURE & calcium-binding region: 2 & 3 & 1.049 & 0.126 \\
\hline INTERPRO & IPR002048:Calcium-binding EF-hand & 4 & 1.399 & 0.148 \\
\hline UP_SEQ_FEATURE & calcium-binding region: 1 & 3 & 1.049 & 0.157 \\
\hline SMART & SM00054:EFh & 4 & 1.399 & 0.187 \\
\hline UP_SEQ_FEATURE & domain:EF-hand 1 & 3 & 1.049 & 0.258 \\
\hline UP_SEQ_FEATURE & domain:EF-hand 2 & 3 & 1.049 & 0.258 \\
\hline INTERPRO & IPR018248:EF hand & 3 & 1.049 & 0.333 \\
\hline Cluster 10 & Enrichment Score: 0.668 & Embryonic development & & \\
\hline Category & Term & Count & $\%$ & PValue \\
\hline GOTERM_BP_FAT & GO:0001824 blastocyst development & 3 & 1.049 & 0.082 \\
\hline GOTERM_BP_FAT & GO:0001701 in utero embryonic development & 4 & 1.399 & 0.165 \\
\hline GOTERM_BP_FAT & GO:0043009 chordate embryonic development & 4 & 1.399 & 0.397 \\
\hline GOTERM_BP_FAT & GO:0009792 embryonic development ending in birth or egg hatching & 4 & 1.399 & 0.400 \\
\hline Cluster 11 & Enrichment Score: 0.565 & Cardiomyopathy & & \\
\hline Category & Term & Count & $\%$ & PValue \\
\hline KEGG_PATHWAY & bta05412:Arrhythmogenic right ventricular cardiomyopathy (ARVC) & 3 & 1.049 & 0.240 \\
\hline KEGG_PATHWAY & bta05410:Hypertrophic cardiomyopathy (HCM) & 3 & 1.049 & 0.277 \\
\hline KEGG_PATHWAY & bta05414:Dilated cardiomyopathy & 3 & 1.049 & 0.304 \\
\hline Cluster 12 & Enrichment Score: 0.519 & Phosphorylation & & \\
\hline Category & Term & Count & $\%$ & PValue \\
\hline GOTERM_MF_FAT & GO:0004672 protein kinase activity & 9 & 3.147 & 0.213 \\
\hline GOTERM_BP_FAT & GO:0006468 protein amino acid phosphorylation & 9 & 3.147 & 0.291 \\
\hline GOTERM_BP_FAT & GO:0016310 phosphorylation & 9 & 3.147 & 0.447 \\
\hline
\end{tabular}

